\title{
EFT triangles in the same-sign $W W$ scattering process at the HL-LHC and HE-LHC
}

\author{
Geetanjali Chaudhary $^{1}$, Jan Kalinowski ${ }^{2}$, Manjit Kaur ${ }^{1}$, Paweł Kozów ${ }^{2,3, a}$, Kaur Sandeep ${ }^{1}$, Michał Szleper ${ }^{4}$, \\ Sławomir Tkaczyk ${ }^{5}$ \\ ${ }^{1}$ Panjab University, Chandigarh 160014, India \\ 2 Faculty of Physics, University of Warsaw, Ul. Pasteura 5, 02-093 Warsaw, Poland \\ ${ }^{3}$ CAFPE and Departamento de Física Teórica y del Cosmos, Universidad de Granada, Campus de Fuentenueva, 18071 Granada, Spain \\ ${ }^{4}$ High Energy Physics Department, National Center for Nuclear Research, Ul. Pasteura 7, 02-093 Warsaw, Poland \\ ${ }^{5}$ Fermi National Accelerator Laboratory, Batavia, IL 60510, USA
}

Received: 20 July 2019 / Accepted: 7 February 2020 / Published online: 27 February 2020

(C) The Author(s) 2020

\begin{abstract}
We investigate the Beyond Standard Model discovery potential in the framework of the effective field theory (EFT) for the same-sign $W W$ scattering process in purely leptonic $W$ decay modes at the High-Luminosity and HighEnergy phases of the Large Hadron Collider (LHC). The goal of this paper is to examine the applicability of the EFT approach, with one dimension- 8 operator varied at a time, to describe a hypothetical new physics signal in the $W W W W$ quartic coupling. In the considered process there is no experimental handle on the $W W$ invariant mass, and it has previously been shown that the discovery potential at $14 \mathrm{TeV}$ is rather slim. In this paper we report the results calculated for a $27 \mathrm{TeV}$ machine and compare them with the discovery potential obtained at $14 \mathrm{TeV}$. We find that while the respective discovery regions shift to lower values of the Wilson coefficients, the overall discovery potential of this procedure does not get significantly larger with a higher beam energy.
\end{abstract}

\section{Introduction}

The Large Hadron Collider (LHC) has completed data taking for Run II. While a lot of collected data still awaits to be analyzed, no physics beyond the standard model (BSM) has been announced until now. The lack of direct indications for the presence of new physics (NP) makes indirect searches more interesting. The High Luminosity LHC (HLLHC) upgrade will eventually collect an integrated luminosity of $3 \mathrm{ab}^{-1}$ of data in $p p$ collisions at a center-of-mass (c.o.m.) energy of $14 \mathrm{TeV}$, which should maximize the LHC potential to uncover new phenomena. It may however well

a e-mail: pkozow@ fuw.edu.pl (corresponding author) be that the NP degrees of freedom are at higher masses making it difficult at the LHC to identify experimentally new particles, or new paradigms. These considerations have been driving, in the last few years, intense activity worldwide to assess the future of collider experiments beyond the HLLHC. Several proposals and studies have been performed. The prospects of pushing the LHC program further with the LHC tunnel and the whole CERN infrastructure, together with future magnet technology, is an exciting possibility that could push the energy up into an unexplored region with the 27 TeV High Energy LHC (HE-LHC), that could collect an integrated luminosity of $15 \mathrm{ab}^{-1}$.

Precision measurements provide an important tool to search for heavy BSM dynamics, associated with mass scales beyond the LHC direct energy reach, exploiting the fact that such dynamics can still have an impact on processes at smaller energy, via virtual effects. In this context the well-established framework of effective field theories (EFTs) allows to systematically parameterize BSM effects and elucidate how they modify SM processes. The BSM contributions are effectively parametrized in terms of higher dimension operators $\mathcal{O}_{i}^{(n)}$, with some effective couplings $C_{i}^{(n)}$ suppressed by appropriate powers of an unknown energy scale $\Lambda$ at which new physics sets in,

$\mathcal{L}=\mathcal{L}_{S M}+\Sigma_{i} \frac{C_{i}^{(6)}}{\Lambda^{2}} \mathcal{O}_{i}^{(6)}+\Sigma_{i} \frac{C_{i}^{(8)}}{\Lambda^{4}} \mathcal{O}_{i}^{(8)}+\cdots$,

where the superscript $n=6,8$ indicates the dimensionality of the corresponding operator. Following the usual notation, we introduce a set of Wilson coefficients $f_{i}^{(n)}$, defined as

$f_{i}^{(6)}=\frac{C_{i}^{(6)}}{\Lambda^{2}}, \quad f_{i}^{(8)}=\frac{C_{i}^{(8)}}{\Lambda^{4}}, \ldots$, 
which are free parameters since neither $C_{i}^{(n)}$ nor $\Lambda$ of the full theory are known. Equation (1.1) represents in principle an infinite and model-independent parametrization of possible BSM effects, valid by construction up to the cutoff value $\Lambda$ in the energy scale of the studied process. The EFT approach is based on the assumption that a limited number of chosen operators contribute to the given process in the studied energy range. In particular, vector boson scattering (VBS) processes are widely recognized as the best laboratory to study the operators which modify only the $V V V V$ quartic couplings. In the SM EFT framework these operators start at dimension-8. The usual ATLAS and CMS procedure in VBS data analyses to date involves testing one such dimension- 8 operator at a time. The goal of this paper is to investigate the sensitivity of this approach by identifying for each operator the corresponding reach in the $\left(f_{i}, \Lambda\right)$ plane at the HE-LHC.

In a recent paper [1] the physics potential of the single dimension-8 operator EFT approach has been tested on a hypothetical new physics signal observed in the same-sign $W W$ scattering process at the HL-LHC. The analysis was focused on the "gold-plated" purely leptonic $W$ decay modes:

$p p \rightarrow 2 j e t s+l^{+} v+l^{\prime+} \bar{v}^{\prime}$

where $l$ and $l^{\prime}$ stand for any combination of electrons and muons. In this process the $M_{W W}$ invariant mass cannot be reconstructed experimentally on the event-by-event basis, leading to a restricted space in the $\left(f_{i}, \Lambda\right)$ plane (the "EFT triangle") for which the single-operator EFT description of the data is viable. In this note we extend such investigations to the HE-LHC energy and expected luminosity domain.

The paper is organized as follows. In Sect. 2 we remind the concept of "EFT triangles". In Sect. 3. we detail our procedure of event simulation and subsequent treatment of generated events. In Sect. 4 we summarize our findings and conclude. Three appendices contain supplementary material. In the first appendix the definitions of dimension- 8 operators are recalled. In Appendix B we argue that qualitative features of the full $W W$ scattering process, including off-shell effects, can be inferred from considering the on-shell $W W$ scattering amplitudes and we discuss helicity amplitudes after adding the higher dimension operators. In the third appendix we address the question of what values of BSM couplings can be drawn from the discovery regions.

\section{EFT triangles}

Since the truncation of the expansion in Eq. (1.1) introduces model dependence, in Ref. [1] the concept of "EFT models" has been introduced where they are defined by the choice of operators $\mathcal{O}_{i}^{(n)}$ and the values of Wilson coefficients $f_{i}^{(n)}$. The
EFT description is valid up to a cutoff energy $\Lambda$ at which new states are expected to appear; the cutoff value is unknown a priori. However, in the presence of higher dimension operators the scattering amplitudes grow with energy and eventually break the perturbative unitarity limit $M^{U}$. The condition $\Lambda<M^{U}\left(f_{i}\right)$ defines the upper bound on the range of possible values of $\Lambda$ as a function of $f_{i}$.

A specific feature of the process in Eq. (1.3) is that the scale $M \equiv M_{W W}$, i.e., the invariant mass of the scattered $W W$ bosons, is not experimentally accessible, making it impossible to properly apply the cutoff $\Lambda$ on the data. Any BSM signal, $S$, is defined as the deviation from the SM prediction in the differential distributions $d \sigma / d x_{i}$ of some observable $x_{i}$.

$\frac{d S}{d x_{i}}=\left(\frac{d \sigma}{d x_{i}}\right)^{B S M}-\left(\frac{d \sigma}{d x_{i}}\right)^{S M}$.

Any collected data sample will in general be a sum of the contributions from $M<\Lambda$ and $M>\Lambda$ (unless $\Lambda$ happens to be out of kinematic reach). To cope with events with $M>\Lambda$, different solutions have been advocated in the literature, e.g.: from discarding these events at the level of simulation, to invoking unitarization procedures (usually assuming $\Lambda=$ $M^{U}$ ) [2,3], to ignoring the cutoff altogether. Any of the above prescriptions is related to additional arbitrariness of choices and therefore affects the physics interpretation of the results. Genuine data interpretation in the EFT language requires its successful description without any additional assumptions as to the nature of BSM physics at the scale above $\Lambda$. This is only possible if the bulk of the total observed BSM signal originates indeed from the EFT-controlled range.

The EFT-controlled signal reads:

$$
\begin{aligned}
\left(\frac{d \sigma}{d x_{i}}\right)^{E F T}= & \int_{2 M_{W}}^{\Lambda}\left(\frac{d^{2} \sigma}{d x_{i} d M}\right)^{E F T} d M \\
& +\int_{\Lambda}^{M_{\max }}\left(\frac{d^{2} \sigma}{d x_{i} d M}\right)^{S M} d M,
\end{aligned}
$$

Here $M_{\max }$ is the kinematic limit of the $W W$ invariant mass. Eq. (2.2) defines signal coming uniquely from the "EFT model" in its range of validity and assumes only the SM contribution in the region $M>\Lambda$.

The additional contribution from the region above $\Lambda$ may enhance the signal, but it may also preclude proper description of the data within the EFT. The total BSM signal can be estimated without detailed knowledge of the UV completion from the expected asymptotic behavior for $M \rightarrow \infty$, i.e., by assuming that all the helicity amplitudes above $\Lambda$ remain constant at their respective values they reach at $\Lambda$ (hence superscript $A=$ const):

$$
\left(\frac{d \sigma}{d x_{i}}\right)^{B S M}=\int_{2 M_{W}}^{\Lambda}\left(\frac{d^{2} \sigma}{d x_{i} d M}\right)^{E F T} d M
$$




$$
+\int_{\Lambda}^{M_{\max }}\left(\frac{d^{2} \sigma}{d x_{i} d M}\right)^{A=\text { const }} d M
$$

For every value of $\Lambda$, BSM observability imposes some minimum value of $f_{i}$ for which the total BSM signal defined by Eq. (2.3) has enough statistical significance, $5 \sigma$ in our example. Successful description in the EFT framework imposes some maximum value of $f_{i}$ such that signal estimates computed from Eqs. (2.2) and (2.3) remain statistically consistent, e.g., within $2 \sigma$. The "EFT triangle" is the region in the $\left(f_{i}, \Lambda\right)$ plane for which a statistically significant BSM signal can be successfully described with a chosen higher dimension operator $\mathcal{O}_{i}$. It is bounded from three sides:

- from above by the unitarity limit $M^{U}\left(f_{i}\right)$,

- from the left by the signal significance of $5 \sigma$, computed according to Eq. (2.3),

- and from the right by the consistency within $2 \sigma$ with Eq. (2.2).

In the HL-LHC case, for all the individual dimension8 operators that affect the $W W W W$ quartic coupling such triangles were found to be rather narrow or even entirely empty (for $\mathcal{O}_{S 1}$ ) [1]. In this paper we extend this analysis to the HE-LHC case, in an attempt to verify if an increased beam energy and integrated luminosity will translate into larger EFT triangles.

Throughout this work we follow the MadGraph convention for the definition of dimension-8 operators (implemented therein via public UFO files [4]), in which the field strength tensors $W_{\mu \nu} \equiv \frac{i}{2} g \tau^{i}\left(\partial_{\mu} W_{\nu}^{i}-\partial_{\nu} W_{\mu}^{i}+g \epsilon_{i j k} W_{\mu}^{j} W_{\nu}^{k}\right)$ are replaced with $\hat{W}_{\mu \nu} \equiv \frac{1}{i g} W_{\mu \nu}$. Such conversion factors are equivalent to absorbing the electroweak coupling constants $g$, each one explicitly factored out for each occurrence of the field stress tensor, in the effective couplings $C_{i}$. For the reader's convenience, Appendix A lists dimension- 8 operators; more details can be found in Ref. [5].

\section{Analysis}

In this section we present a generator-level study aimed at finding the EFT triangles for the individual $n=8$ operators at the HE-LHC. Event samples of the process $p p \rightarrow$ $j j \mu^{+} \mu^{+} \nu v$ at $27 \mathrm{TeV}$ were generated for each $n=8$ operator $\mathcal{O}_{i}$ that modifies the $W W W W$ quartic coupling, $i=S 0, S 1$ (so called scalar operators), $T 0, T 1, T 2$ (transverse), and $M 0, M 1, M 6, M 7$ (mixed $^{1}$ ones). Generation has been done at LO using MadGraph5_aMC@NLO v5.2.6.2

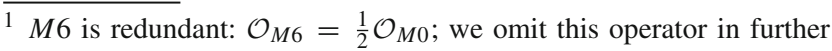
analysis.
}

generator [6], with the appropriate UFO files containing additional vertices involving the desired $n=8$ operators. A scan of $f_{i}$ values for each operator was made using the MadGraph reweight command, including $f_{i}=0$ to represent the SM case. The Pythia package v6.4.1.9 [7] was used for hadronization as well as initial and final state radiation processes. Unitarity limits were determined using the VBFNLO [8,9] calculator v1.4.0, after applying appropriate Wilson coefficients conversion factors. Cross sections at the output of MadGraph were multiplied by a factor 4 to account for all the lepton (electron and/or muon) combinations in the final state (in this work only positively charged leptons are taken into account, although the same analysis can be done for the negative charges). Only signal samples were generated and the SM case was treated as irreducible background in the study of possible BSM effects.

The applied analysis chain to the events generated for HE-LHC is a carbon copy of the one described in detail in Ref. [1], here we only briefly outline the main points, with special emphasis on the differences. Standard VBS selection criteria were applied, namely we require at least two reconstructed jets and exactly two leptons (muons or electrons) satisfying the following conditions: $M_{j j}>500 \mathrm{GeV}$, $\Delta \eta_{j j}>2.5, p_{T}^{j}>30 \mathrm{GeV},\left|\eta_{j}\right|<5, p_{T}^{l}>25 \mathrm{GeV}$ and $\left|\eta_{l}\right|<2.5$. Although we have not optimized our selection criteria for $27 \mathrm{TeV}$, one can safely suppose they will not be much different. A detailed optimization will be done with full knowledge of the future detector geometry. Like before, the total BSM signal is estimated according to Eq. (2.3) by suppressing the high-mass tail above the calculated value of $\Lambda$. This is achieved by applying an additional weight of the form $\left(\Lambda / M_{W W}\right)^{4}$ to each generated event in this region. The EFT-controlled signal is calculated according to Eq. (2.2) by replacing the generated high-mass tail with the one expected in the SM (known as "clipping method"). Signal significances are computed as the square root of a $\chi^{2}$ resulting from a binby-bin comparison of the event yields, with statistical errors such as expected from the data at $3 \mathrm{ab}^{-1}$, in the distribution of the most sensitive kinematic variables. Compared to the $14 \mathrm{TeV}$ analysis, the binning of histograms was changed so that in the highest bin the SM prediction normalized to 3/ab is still between 2 and 3 events. In Fig. 1 as an example shown are distributions of four chosen variables:

- invariant mass of two leptons $M_{l l}$,

- ratio of transverse momenta of leptons and jets $R_{p_{T}} \equiv$ $p_{T}^{l 1} p_{T}^{l 2} /\left(p_{T}^{j 1} p_{T}^{j 2}\right)$,

- $M_{o 1} \equiv \sqrt{\left(\left|\mathbf{p}_{T}^{l 1}\right|+\left|\mathbf{p}_{T}^{l 2}\right|+\left|\mathbf{p}_{T}^{\text {miss }}\right|\right)^{2}-\left(\mathbf{p}_{T}^{l 1}+\mathbf{p}_{T}^{l 2}+\mathbf{p}_{T}^{\text {miss }}\right)^{2}}$

- and the (true) invariant mass $M_{W W}$

in the SM and in the case $f_{M 1}=0.2 \mathrm{TeV}^{-4}$ and $\Lambda=4.9$ $\mathrm{TeV}$. As it was for $14 \mathrm{TeV}$, we found $R_{p_{T}}$ to be the most 

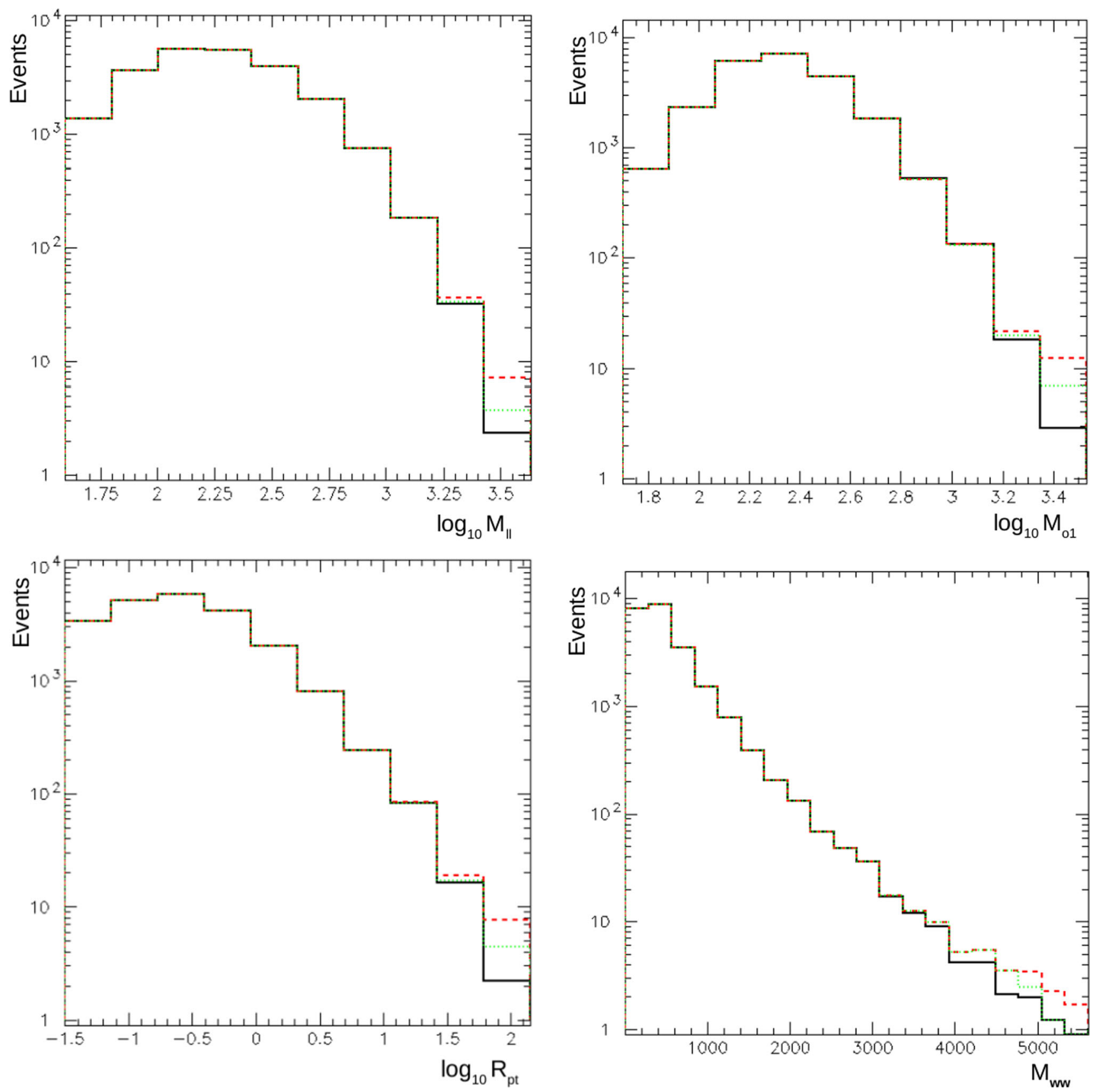

Fig. 1 Typical examples of kinematic distributions used for the assessment of BSM signal significances. Shown are the distributions of $M_{l l}$, $M_{o 1}$ and $R_{p T}$ (in $\log$ scale): in the Standard Model (black), with $f_{M 1}=0.2 \mathrm{TeV}^{-4}$ and the high- $M_{W W}$ tail treatment according to Eq. (2.3) (red), and with $f_{M 1}=0.2 \mathrm{TeV}^{-4}$ and the high- $M_{W W}$ tail

treatment according to Eq. (2.2) (green). In addition the lower-right plot shows the distribution in the invariant mass of the WW system, $M_{W W}$ with $f_{M 1}=0.2 \mathrm{TeV}^{-4}$. In all the plots the scale $\Lambda$ was chosen as: $\Lambda=M^{U}=4.9 \mathrm{TeV}$. Assumed is $\sqrt{s}=27 \mathrm{TeV}$ and an integrated luminosity of $3 \mathrm{ab}^{-1}$

sensitive kinematic variable for $\mathcal{O}_{S 0}$ and $\mathcal{O}_{S 1}$, and $M_{o 1}$ for the remaining operators.

As the unitarity limit we take always the lower of the two values between on-shell $W^{+} W^{+}$and on-shell $W^{+} W^{-}$ scattering, calculated from T-matrix diagonalization in the helicity space. Indeed, both processes probe the same quartic

coupling and are governed by the same Wilson coefficients, as further explained in Appendix B.3.

Figure 2 shows the results for the individual operators $S 0, S 1, T 0, T 1, T 2, M 0, M 1$ and $M 7$, in comparison with results at $14 \mathrm{TeV}$ (for positive $f$ values). Not unexpectedly, all the triangles are shifted to lower $f$ values compared 

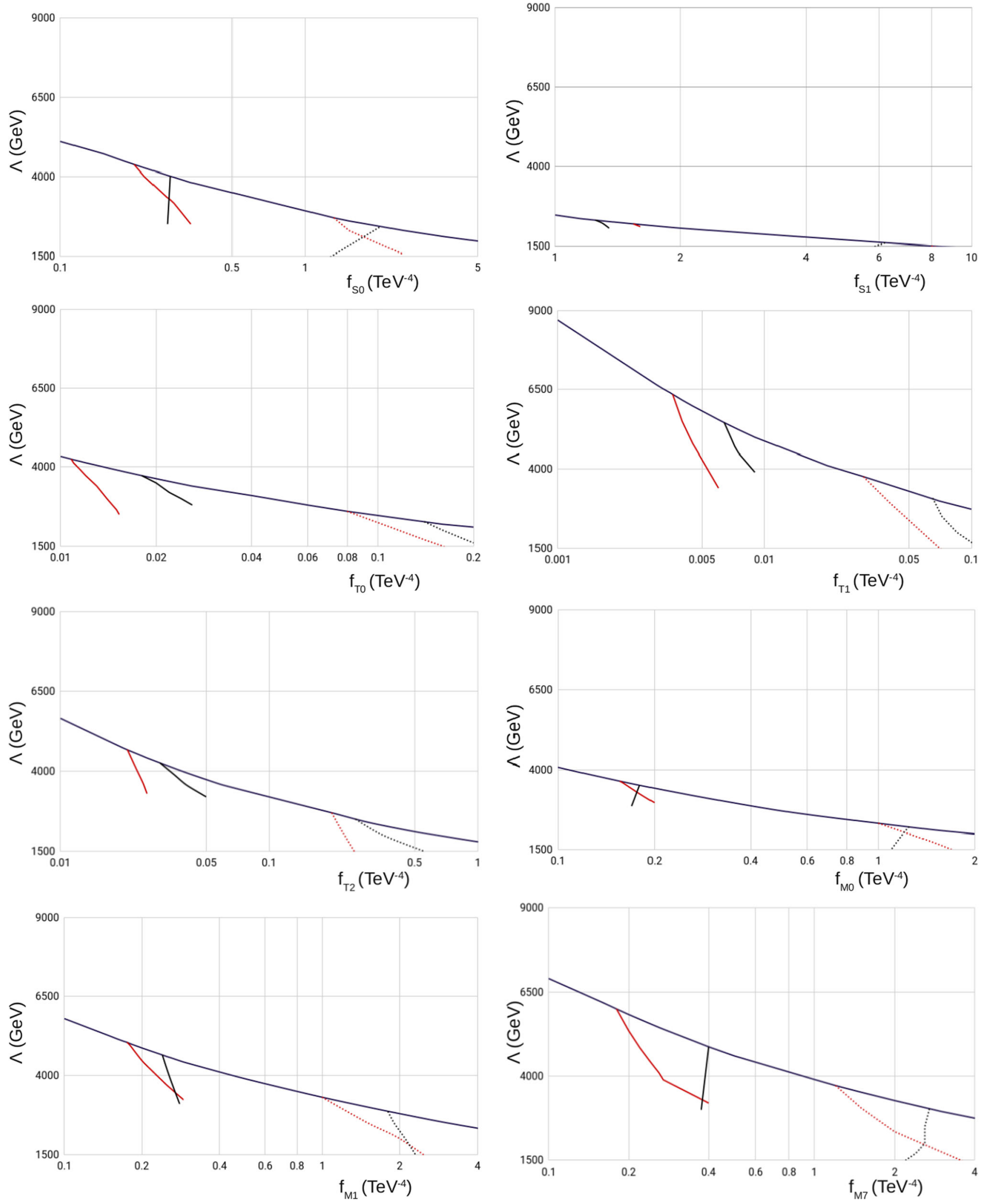

Fig. 2 Regions in the $\Lambda$ vs $f$ (positive $f$ values) space for dimension8 operators in which a $5 \sigma$ BSM signal can be observed and the EFT is applicable. The unitarity limit is shown in blue; the lower limits

for a $5 \sigma$ signal significance from Eq. (2.3) (red) and the upper limit on $2 \sigma$ EFT consistency (black). The solid (dotted) lines correspond to $\sqrt{s}=27$ (14) TeV. Assumed is the integrated luminosity of $3 \mathrm{ab}^{-1}$ 

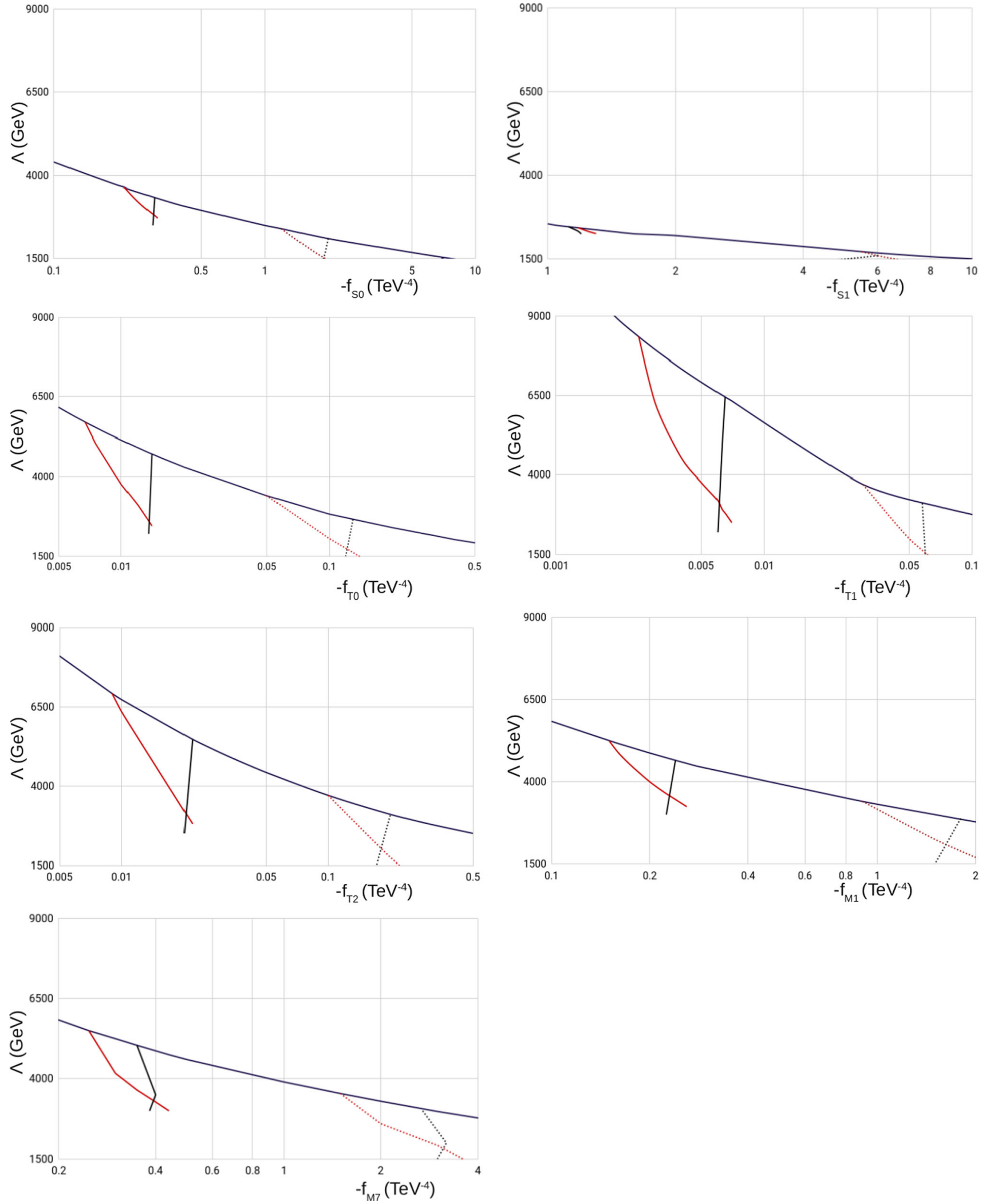

Fig. 3 Regions in the $\Lambda$ vs $f$ (negative $f$ values) space for dimension8 operators in which a $5 \sigma$ BSM signal can be observed and the EFT is applicable. The unitarity limit is shown in blue; the lower limits

for a $5 \sigma$ signal significance from Eq. (2.3) (red) and the upper limit on $2 \sigma$ EFT consistency (black). The solid (dotted) lines correspond to $\sqrt{s}=27$ (14) TeV. Assumed is the integrated luminosity of $3 \mathrm{ab}^{-1}$ 


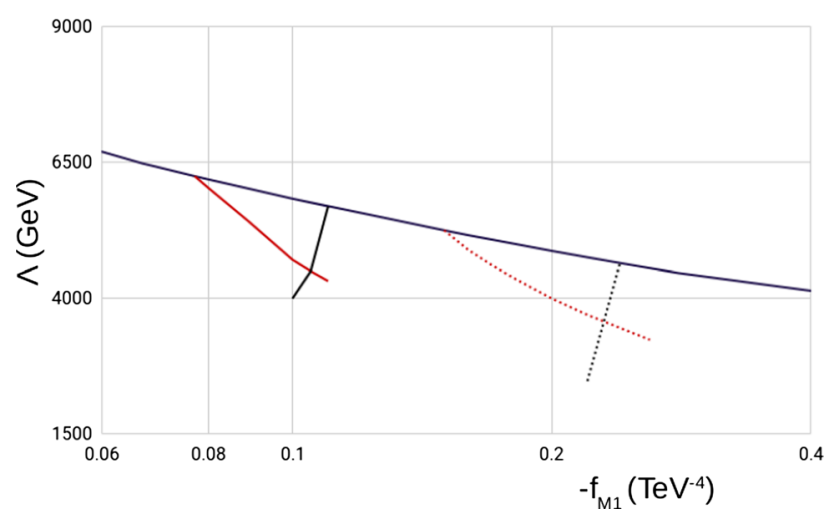

Fig. 4 Regions in the $\Lambda$ vs $f$ (negative $f$ values) space for $M 1$ operator in which a $5 \sigma$ BSM signal can be observed and the EFT is applicable. The unitarity limit is shown in blue; the lower limits for a $5 \sigma$ signal significance from Eq. (2.3) (red) and the upper limit on $2 \sigma$ EFT consistency (black). The solid (dotted) lines correspond to $15 \mathrm{ab}^{-1}\left(3 \mathrm{ab}^{-1}\right)$. Assumed is $\sqrt{s}=27 \mathrm{TeV}$

to $14 \mathrm{TeV}$, the shift being as large as almost an order of magnitude. However, the total area of the triangles does not get significantly larger as we increase the energy. This is because the EFT consistency criterion pushes the effective upper limits on $f$ in a similar manner as does the BSM observability criterion for the lower limits. Overall, the shapes and sizes of all the EFT triangles are remarkably similar for $27 \mathrm{TeV}$ as for $14 \mathrm{TeV}$, only their respective positions differ.

Figure 3 shows the respective results for negative $f$ values of $S 0, S 1, T 0, T 1, T 2, M 1$ and $M 7$. Here exactly the same observations can be made again. The negative $f$ values of $M 0$ look virtually identical to their positive counterparts, since for these operators the SM-BSM interference term in the total amplitude calculation is practically negligible (see the Appendix for details), and so we do not show them here. There is no triangle at all for $S 1$, for which the overall lower limit for BSM observability is about $1.2 \mathrm{TeV}^{-4}$ and the upper limit for EFT consistency is $1.4 \mathrm{TeV}^{-4}$. Here as well we observe a similar behavior as for $14 \mathrm{TeV}$.

Both analyses, at 14 and $27 \mathrm{TeV}$, were done at the generator level. Reducible backgrounds were not simulated, since they are known to be strongly detector dependent (for the different compositions of reducible background as measured at $13 \mathrm{TeV}$ by CMS and ATLAS, see Figs. 2-a and 2-b in Ref. [10] and Fig. 3 in Ref. [11], respectively, where it has been found that 'non-prompt' leptons (from hadron decay and fakes) is the largest reducible background and WZ is small in CMS, while in ATLAS WZ is the largest background and 'nonprompt' is small). Such simulations have to be carried by each experiment for its specific detector performances. For a realistic estimate of the sensitivity limits to new physics effects the reader is referred to literature, e.g., Ref. [12]. Full detector simulation will move the EFT triangles to larger
Wilson coefficients, but it cannot make them larger, hence all our conclusions still hold.

For the sake of a convenient comparison between the respective results at two different $p p$ beam energies, in the bulk of this study we have always assumed the same integrated luminosity of $3 \mathrm{ab}^{-1}$ for both cases. This number is appropriate for the HL-LHC stage, but underestimates the expected statistical power of the HE-LHC. However, it is trivial to recalculate all the results to $15 / \mathrm{ab}$ in order to get the true expected discovery reach of the HE-LHC, taking into account its actual expected luminosity. An increase of statistics by a factor 5 will lead to a further shift of all the EFT triangles by a factor close to $\sqrt{5}$, both in the $5 \sigma$ discovery and the $2 \sigma$ consistency curves (in fact, somewhat less than that because of non-linear dependence of the BSM signal on the value of the individual Wilson coefficients). It will not significantly change either the shape nor the size of triangles. A comparison of results calculated for the same $p p$ beam energy of $27 \mathrm{TeV}$ and two different integrated luminosities is exemplified for the $M 1$ operator in Fig. 4.

Our simple procedure to suppress the high-mass tails by applying a $\left(\Lambda / M_{W W}\right)^{4}$ weight to events generated above the scale of $\Lambda$ works reasonably well in the vicinity of the unitarity limit. In this region it produces a tail falling approximately like $1 / M_{W W}^{2}$, which is the expected asymptotic (i.e., for $M_{W W}>>\Lambda$ ) behavior of the total cross section after regularization. It nonetheless becomes too strong as we go to $\Lambda<<M^{U}$, where the total cross section is still dominated by the SM contribution which does not require any further suppression. Moreover, for low values of $\Lambda$ the tail itself becomes large, leading to large uncertainties due to the details of its modeling. We have discontinued the curves in Figs. 2 and 3 below the values at which we find the method lead to the unphysical result of signal being suppressed below the SM level itself. For this reason the EFT triangles for $T 0, T 1$ and $T 2$ do not close. For the remaining cases, however, they are completely contained in the region where our simple method is still viable.

According to Ref. [13], full NLO (EW + QCD) corrections to the SM same-sign WW scattering process at $13 \mathrm{TeV}$ lower the total cross section within a fiducial volume defined by standard VBS cuts by $17 \%$; the relative effect increases in the high invariant mass region. Corresponding corrections at $27 \mathrm{TeV}$ were reported in [14]. They are relatively a few percent larger than in the former case. Therefore, accounting for higher-order corrections is well motivated in further more dedicated studies.

Positivity constraints applied to VBS, derived e.g. in Ref. [15], require that certain linear combinations of Wilson coefficients of dimension- 8 operators to be positive definite. Since we choose one operator at a time, the bounds would determine what signs of the Wilson coefficients should be 
considered for each operator. Therefore accounting for these bounds using our results is straightforward.

\section{Conclusion and outlook}

Although an increase of the LHC energy vastly improves the sensitivity to new physics effects in VBS processes, the question of EFT applicability is a different one and cannot be solved by changing the energy. The same-sign $W W$ process with its purely leptonic $W$ decays is often considered "goldplated" due to its relatively good signal to background ratio, but the lack of experimental access to the $W W$ invariant mass poses a severe problem in describing the data in terms of the EFT. Despite reasonable sensitivity to BSM effects, such effects if observed will most likely not be possible to interpret using the data from this process alone and applying the usual framework of testing one dimension-8 operator at a time. This conclusion holds regardless of the actual proton beam energy.

The present results reinforce the former conclusion that future VBS data analysis, both at the LHC experiments as well as future proton-proton colliders, should evolve in the direction of multidimensional fits with many higher dimension operators varied at a time. This in turn may require global simultaneous fits to many processes (including $W Z, Z Z$ and semi-leptonic $W V$, if not other processes) to help disentangle the correlations between signals originating from different operators. Independently of the goal and results of this paper, one always has to remember that the approach of varying one operator at-a-time is not the most appropriate in any case - such a procedure breaks the model independence of the EFT description and therefore EFT requires ultimately a global approach.

Helpful in disentangling the effects of different operators may be also the polarizations of the outgoing $W$ bosons, as different operator subsets, $\mathrm{S}, \mathrm{T}$ and $\mathrm{M}$, affect different polarizations. There are new theoretical ideas how to project the total VBS cross sections onto individual polarizations without invoking the rather crude $W$ on-shell approximation [16]. $W W$ polarizations can be extracted from the data by fitting simulated templates of the corresponding polarized distributions. Unfortunately, purely leptonic $W W$ decays do not offer the possibility to reconstruct the $W$ decay angle, which is the only strictly model-independent signature of $W$ polarization. While many other distributions exhibit qualitative differences between the different polarizations, they are usually also strongly model-dependent. Consequently, SM templates cannot be used in the BSM case without the risk of losing sensitivity to the BSM signal. If, however, a set of observables is identified for which sufficiently modelindependent templates for $W_{L} W_{L}, W_{T} W_{T}$ and $W_{T} W_{L}$ can be constructed, it could vastly improve the perspectives of future VBS data analysis in the framework of the EFT.

Acknowledgements We would like to thank Adam Falkowski and Luca Merlo for valuable comments and discussions. The work of PK is supported by the Spanish MINECO Project FPA2016-78220-C3-1$\mathrm{P}$ (Fondos FEDER) and by National Science Centre, Poland, the PRELUDIUM project under Contract 2018/29/N/ST2/01153. JK was supported in part by the National Science Centre, Poland, the HARMONIA project under contract UMO-2015/18/M/ST2/00518 (2016-2020). JK and MS were partly supported by the COST Action CA15108 and are grateful to all the members of the Action for inspiring discussions. ST is supported by Fermi Research Alliance, LLC under Contract No. DeAC02-07CH11359 with the United States Department of Energy.

Data Availability Statement This manuscript has no associated data or the data will not be deposited. [Authors' comment: The datasets generated during and/or analysed during the current study are available from the corresponding author on reasonable request.]

Open Access This article is licensed under a Creative Commons Attribution 4.0 International License, which permits use, sharing, adaptation, distribution and reproduction in any medium or format, as long as you give appropriate credit to the original author(s) and the source, provide a link to the Creative Commons licence, and indicate if changes were made. The images or other third party material in this article are included in the article's Creative Commons licence, unless indicated otherwise in a credit line to the material. If material is not included in the article's Creative Commons licence and your intended use is not permitted by statutory regulation or exceeds the permitted use, you will need to obtain permission directly from the copyright holder. To view a copy of this licence, visit http://creativecomm ons.org/licenses/by/4.0/.

Funded by SCOAP ${ }^{3}$.

\section{A Dimension 8 operators}

The following dimension eight operators contribute to the $W W W W$ vertex, without affecting tri-linear couplings:

$$
\begin{aligned}
\mathcal{O}_{S 0} & =\left[\left(D_{\mu} \Phi\right)^{\dagger} D_{\nu} \Phi\right] \times\left[\left(D^{\mu} \Phi\right)^{\dagger} D^{\nu} \Phi\right], \\
\mathcal{O}_{S 1} & =\left[\left(D_{\mu} \Phi\right)^{\dagger} D^{\mu} \Phi\right] \times\left[\left(D_{\nu} \Phi\right)^{\dagger} D^{\nu} \Phi\right], \\
\mathcal{O}_{M 0} & =\operatorname{Tr}\left[W_{\mu \nu} W^{\mu \nu}\right] \times\left[\left(D_{\beta} \Phi\right)^{\dagger} D^{\beta} \Phi\right], \\
\mathcal{O}_{M 1} & =\operatorname{Tr}\left[W_{\mu \nu} W^{\nu \beta}\right] \times\left[\left(D_{\beta} \Phi\right)^{\dagger} D^{\mu} \Phi\right], \\
\mathcal{O}_{M 7} & =\left[\left(D_{\mu} \Phi\right)^{\dagger} W_{\beta \nu} W^{\beta \mu} D^{\nu} \Phi\right], \\
\mathcal{O}_{T 0} & =\operatorname{Tr}\left[W_{\mu \nu} W^{\mu \nu}\right] \times \operatorname{Tr}\left[W_{\alpha \beta} W^{\alpha \beta}\right], \\
\mathcal{O}_{T 1} & =\operatorname{Tr}\left[W_{\alpha \nu} W^{\mu \beta}\right] \times \operatorname{Tr}\left[W_{\mu \beta} W^{\alpha \nu}\right], \\
\mathcal{O}_{T 2} & =\operatorname{Tr}\left[W_{\alpha \mu} W^{\mu \beta}\right] \times \operatorname{Tr}\left[W_{\beta \nu} W^{\nu \alpha}\right],
\end{aligned}
$$

where $\Phi$ is the Higgs doublet field, the covariant derivative $D_{\mu} \equiv \partial_{\mu}+i \frac{g^{\prime}}{2} B_{\mu}+i g W_{\mu}^{i} \frac{\tau^{i}}{2}$ and the field strength tensor $W_{\mu \nu}=\frac{1}{2} \tau^{i}\left(\partial_{\mu} W_{\nu}^{i}-\partial_{\nu} W_{\mu}^{i}+g \epsilon_{i j k} W_{\mu}^{j} W_{\nu}^{k}\right)$. 


\section{B WW scattering: off-shell versus on-shell}

In this Appendix we investigate what can be said about the VBS subprocess in the full $p p \rightarrow j j l l^{\prime} v_{l} v_{l^{\prime}}$ reaction from the analysis of the on-shell $W W$ scattering process. We start with the discussion of the $W W$ scattering in full $p p$ process, then identify the helicity amplitudes that dominate the highenergy behavior in the presence of dimension- 8 operators and discuss the question of determining the unitarity limits.

\section{B.1 $W W$ scattering in the full $p p$ reaction}

In the physical process $p p \rightarrow j j l l^{\prime} v v_{l^{\prime}}$ the $W$ bosons are offshell. Nevertheless, in this subsection we would like to show that qualitative conclusions on the influence of dimension- 8 operators on the full process can be drawn from the analysis of on-shell $W W$ scattering. To this end, let us employ the identity [16]:

$g_{\mu \nu}+\frac{k_{\mu} k_{\nu}}{M_{W}^{2}}=\sum_{\lambda=1}^{4} \epsilon_{\lambda}^{\mu}(k)\left(\epsilon_{\lambda}^{\nu}(k)\right)^{*}$.

to express the numerator of the off-shell vector boson as a sum over polarization vectors $\epsilon_{\lambda}^{\mu}(k)$. In the frame in which the spatial component of $k_{\mu}$ is in the $z$ direction, $k_{\mu}=(E, 0,0, k)$, the explicit form of each polarization vector reads:

$\epsilon_{-}^{\mu}=\frac{1}{\sqrt{2}}(0,+1-i, 0) \quad$ (left),

$\epsilon_{+}^{\mu}=\frac{1}{\sqrt{2}}(0,-1-i, 0) \quad$ (right),

$\epsilon_{0}^{\mu}=(k, 0,0, E) / \sqrt{k^{2}} \quad$ (longitudinal),

$\epsilon_{A}^{\mu}=(E, 0,0, k) / \sqrt{\frac{k^{2}-M_{W}^{2}}{k^{2} M_{W}^{2}}} \quad$ (auxiliary),

where $k^{2} \equiv k_{\mu} k^{\mu}$. In the on-shell limit $k^{2} \rightarrow M_{W}^{2}$ the auxiliary polarization vanishes and $\epsilon_{0}$ approaches the exact on-shell form of longitudinal polarization. With the help of Eq. (B.1) one can then rewrite each of the $4 \mathrm{~W}$ propagators in each of the diagram that has VBS topology, as

$\frac{-i \sum_{\lambda=1}^{4} \epsilon_{\lambda}^{\mu}\left(\epsilon_{\lambda}^{\nu}\right)^{*}}{k^{2}-M_{W}^{2}}$

Then the parton-level amplitude $q q \rightarrow q q l l^{\prime} v_{l} v_{l}^{\prime}$ with VBS topology can be decomposed as follows

$$
\begin{aligned}
M \equiv & \frac{\sum_{\lambda_{1} \lambda_{2} \lambda_{3} \lambda_{4}} M_{\lambda_{1}}^{q 1} M_{\lambda_{2}}^{q 2} M_{\lambda_{1} \lambda_{2} \lambda_{3} \lambda_{4}}^{W W} M_{\lambda_{3}}^{l 1} M_{\lambda_{4}}^{l 2}}{\left(k_{1}^{2}-M_{W}^{2}\right)\left(k_{2}^{2}-M_{W}^{2}\right)\left(k_{3}^{2}-M_{W}^{2}\right)\left(k_{4}^{2}-M_{W}^{2}\right)}, \\
& \lambda_{i} \in\left\{\epsilon_{-}, \epsilon_{+}, \epsilon_{0}, \epsilon_{A}\right\} .
\end{aligned}
$$

The $M_{\lambda_{i}}^{q i}\left(M_{\lambda_{i}}^{l i}\right)$ terms are the trilinear $q q W(l l W)$ vertices contracted with $\epsilon^{*}(\epsilon)$ of Eq. (B.3), while the $M_{\lambda_{1} \lambda_{2} \lambda_{3} \lambda_{4}}^{W W}$ term is the (off-shell) $W W$ elastic scattering amplitude. The sum over $i$ includes necessarily polarization configurations in which the $W$ polarizations are auxiliary. Now, the effect of dimension-8 operators grows with the scattering energy $M_{W W}>>M_{W}$ and modifies significantly helicity amplitudes so that deviations from the SM behavior become nonnegligible. Since the off-shellness $k_{i}^{2}$ are suppressed dynamically by propagators $1 /\left(k_{i}^{2}-M_{W}^{2}\right)$, in this kinematic limit the scattered vector bosons must be fast, $\left|\mathbf{k}_{\mathbf{i}}\right| \sim E_{i}>>M_{W}$, Therefore in the high $M_{W W}$ region $\epsilon_{0}^{\mu} \sim \epsilon_{A}^{\mu}$ and approach the on-shell form of the longitudinal polarization vector. As a result, the sum in Eq. (B.3) runs effectively over $\epsilon_{i}=\epsilon_{0}, \epsilon_{+}, \epsilon_{-}$and the off-shell helicity amplitude can be approximated by the on-shell one, accounting corrections of order $\sqrt{\left(k^{2}-M_{W}^{2}\right) /\left(k^{2} M_{W}^{2}\right)}$ or $1 / \sqrt{k^{2}}$. Therefore in the following subsections we will discuss in detail the high-energy behavior of on-shell $W W$ scattering in the presence of contributions from dimension- 8 operators and the unitarity bound.

\section{B.2 The on-shell $W W$ scattering and the helicity amplitudes}

Let us consider the elastic on-shell $W^{+} W^{+} \rightarrow W^{+} W^{+}$in the presence of BSM part represented by a single dimension8 operator, as in an "EFT model". The scattering amplitude $i M$ can be written as:

$i M=A_{S M}+A_{B S M}$,

where $A_{S M}$ denotes the SM part and $A_{B S M}$ represents the BSM part that depends on the Wilson coefficients $f_{i}$.

For the on-shell $W$ bosons we choose to work in the helicity basis in which the polarizations are $\epsilon_{i}^{\mu}$ with $i=+,-, 0$. There are in total $3^{4}=81$ helicity amplitudes $i M(i j \rightarrow$ $k l)$ corresponding to helicity configurations $(i j k l)$ in the $W W \rightarrow W W$ scattering process. The total unpolarized onshell $W W$ cross section can schematically be written as:

$$
\begin{array}{r}
\sigma \sim \frac{1}{9} \sum_{i, j, k, l}\left|A_{S M}(i j \rightarrow k l)\right|^{2}+\left(A_{S M}(i j \rightarrow k l)\right. \\
\left.A_{B S M}(i j \rightarrow k l)^{*}+\text { h.c. }\right)+\left|A_{B S M}(i j \rightarrow k l)\right|^{2}
\end{array}
$$

Since there are orders of magnitude differences concerning contributions of different helicity amplitudes to the total cross section it is convenient, using discrete symmetries $\mathcal{P}$ and $\mathcal{T}$ and Bose statistics, to divide 81 polarization amplitudes into classes. Amplitudes from the same class yield the same contribution to (polarized) cross sections. Hence, in practice one can consider a reduced number of 13 independent polarization classes, taking into account their multiplicities when computing the cross section. It turns out that only a few helicity configurations contribute non-negligibly at high 


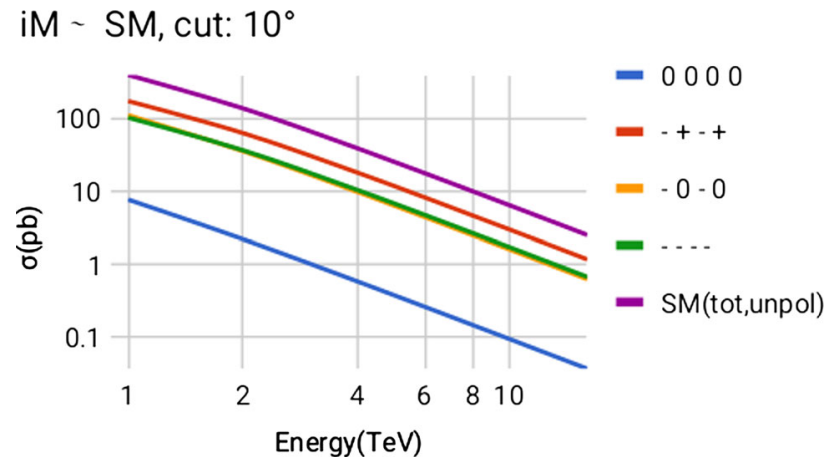

Fig. 5 Contributions of various helicitity configurations (multiplicity taken into account) to the total unpolarized cross section as a function of the center-of-mass collision energy $\left(E_{C M} \equiv \sqrt{s}\right.$, in TeV) in the SM. The total unpolarized cross section is shown in violet

$W W$ scattering energy. We refer to such helicities as saturating helicities.

For the case of the SM the contribution from the saturating helicities to the total unpolarized cross-section is shown in Fig. 5. The four saturating helicity configurations are the only ones whose scattering amplitude is asymptotically constant in energy. The remaining helicity configurations behave asymptotically at most as $1 / s$, hence their contribution is strongly suppressed at large $s=M_{W W}^{2}$. All on-shell $W W$ cross sections are computed with a $10^{\circ}$ cut in the forward and backward scattering regions (which is explicitly written in the plots).

In the presence of $n=8$ operators some of the saturating helicities grow with $s$, maximally as $s^{2}$. The corresponding case for each "EFT model" studied is shown in Figs. 6 and 7 for $f_{i}>0$ and $f_{i}<0$, respectively. In fact, for each "EFT model" there is at least one polarization configuration with the asymptotic $s^{2}$ energy dependence providing dominant contribution to the unpolarized cross section at $M_{W W}=M^{U}$. In particular, in the case of "EFT models" with scalar operators $(S)$ only the amplitude with all $W$ bosons polarized longitudinally grows as $s^{2}$. In the case of transverse operators $(T)$ some amplitudes with all $W$ polarized transversally grow as $s^{2}$, while for the case of mixed operators $(M)$ it happens for amplitudes with two longitudinal and two transverse polarizations. It follows from $D_{\mu} \Phi$ and $W_{\mu \nu}$ building blocks of BSM operators which project mostly on the longitudinal and transverse modes, respectively. It is interesting to notice, however, that for different $S, T$ and $M$ distinct polarization configurations of the outgoing $W$ 's dominate the total cross section at large $M_{W W}$. Measurement of final state $W$ polarizations would give an insight to the dynamics of their interactions.

Since helicity is an observable for the on-shell $W W$ scattering reaction, different helicity configurations do not interfere among themselves. The total unpolarized elastic onshell $W W$ cross sections as a function of the center of mass
$W W$ energy and its dependence on the $f_{i}$ sign is shown in Fig. 8. The sign dependence of the total unpolarized cross section, most visible for $T 0, T 2$ and also present for $T 1, M 1, M 7, S 0, S 1$, is due to the interference terms in Eq. (B.6). More precisely, the dependence on the sign of the $f_{i}$ is determined by the magnitude of SM-BSM terms relative to the $\mathrm{BSM}^{2}$ ones in the region $E \lesssim \Lambda \leq M^{U}$. While there are always $\mathrm{BSM}^{2}$ terms that asymptotically behave as $s^{4} / \Lambda^{8}$, the earlier growth as $\sim s^{2} / \Lambda^{4}$ of the interference terms is not necessarily visible in each of the "EFT models". If the helicity configurations for which the amplitude depends on energy as $s^{2}$ are not among the saturating helicities of the $\mathrm{SM}$, extra suppression factor(s) of $v / \Lambda<<1$ with respect to the opposite case, will be present in the SM-BSM terms. The latter means suppressed sign dependence of the unpolarized cross section, i.e. suppressed interference. It can be inferred from the polarization decomposition plots in Figs. 6, 7 that it is the case for the $M 0$ operator, and indeed in Fig. 8 it is seen that the interference effect is practically invisible for this operator.

Although for the off-shell bosons the helicities are not observable and amplitudes with different helicity configurations may interfere, their interference will be dumped by different structures of fermionic currents to which they are coupled.

\section{B.3 The unitarity bound}

The dominating polarization configurations in the total unpolarized cross section can be read from Figs. 6, 7. However, the helicity combination that determines the $M^{U}$, i.e. that yields strongest unitarity bound, is not necessarily among them. The reason is as follows. The partial wave expansion of helicity amplitude starts with $J_{\min }=\max \left\{\left|\lambda_{1}-\lambda_{2}\right|,\left|\lambda_{3}-\lambda_{4}\right|\right\}$, where $\lambda_{1,2}$ and $\lambda_{3,4}$ correspond initial and final $W$ polarizations, and it is the $J=J_{\min }$ partial wave that yields the strongest unitarity limit. It has been checked that for the same-sign $W W$ helicity amplitude that depends on energy as $s^{2}$ for the case of $M$ operators $J_{\min }=1$, while for the $S$ and $T$ operators $J_{\min }=0$. It would imply then that the unitarity limit for the $M$ operators would be weaker than for $S$ and $T$, especially if only the $J=0$ partial waves were considered. However, the same operators affect both the samesign and opposite-sign $W W$ scattering processes, and both processes should be considered for the determination of the unitarity bounds. In the case of the latter reaction the number of independent helicity configurations is 17 resulting from the fact that one can use all three $\mathcal{C}, \mathcal{P}$ and $\mathcal{T}$ symmetries; however one cannot use the Bose symmetry. In this case for each "EFT model", including the $M$ ones, there exists a helicity configuration that depends on energy as $s^{2}$ and has $J_{\min }=0$. As a result, for the $M$-type "EFT model" the unitarity limit is considerably stronger as compared to the limit 

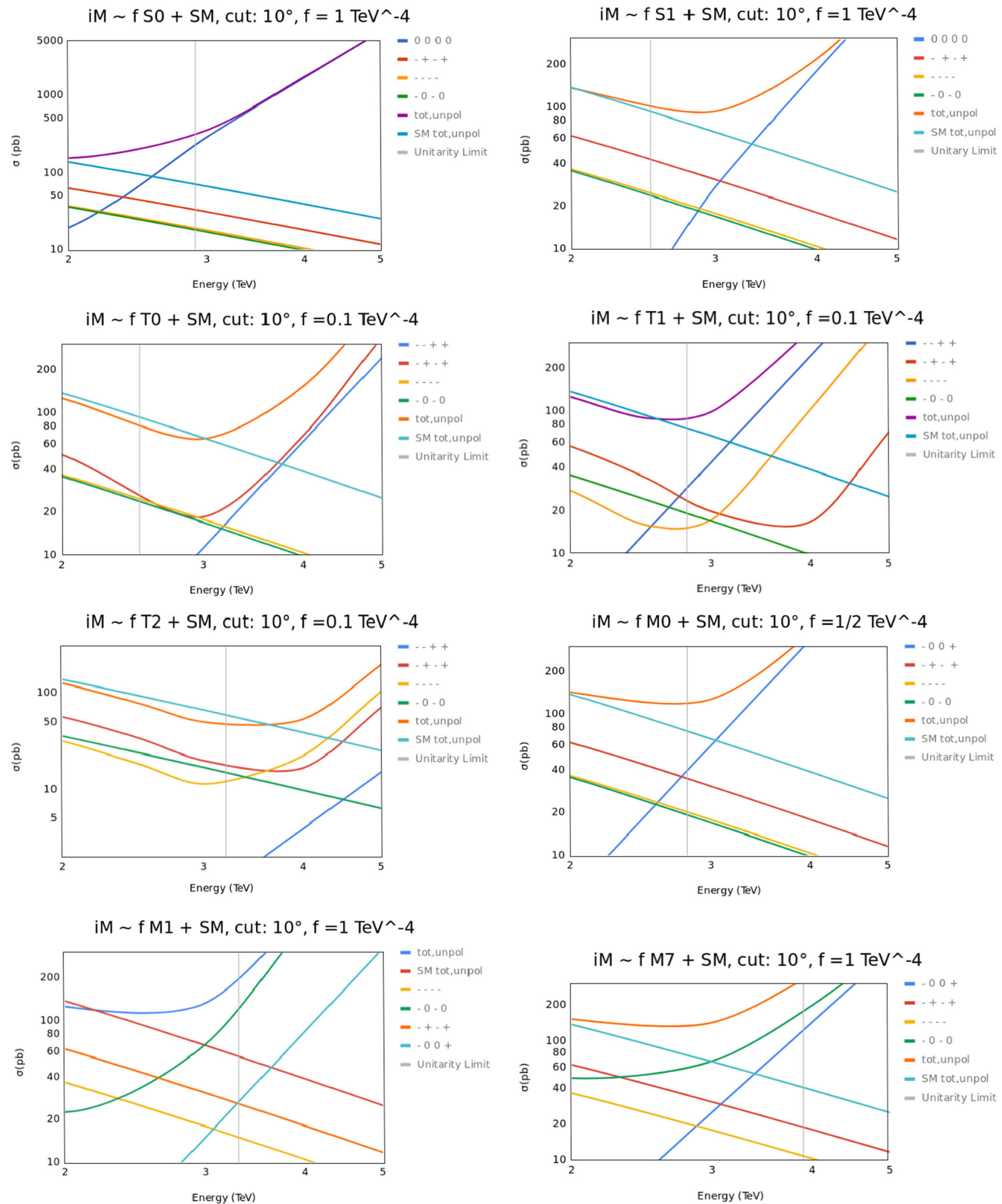

Fig. 6 Contributions of the polarized cross sections (multiplicity taken into account) as functions of the center-of-mass collision energy $\left(E_{C M} \equiv \sqrt{s}\right.$, in $\mathrm{TeV}$ ) for chosen values of $f_{i}>0$. The remaining
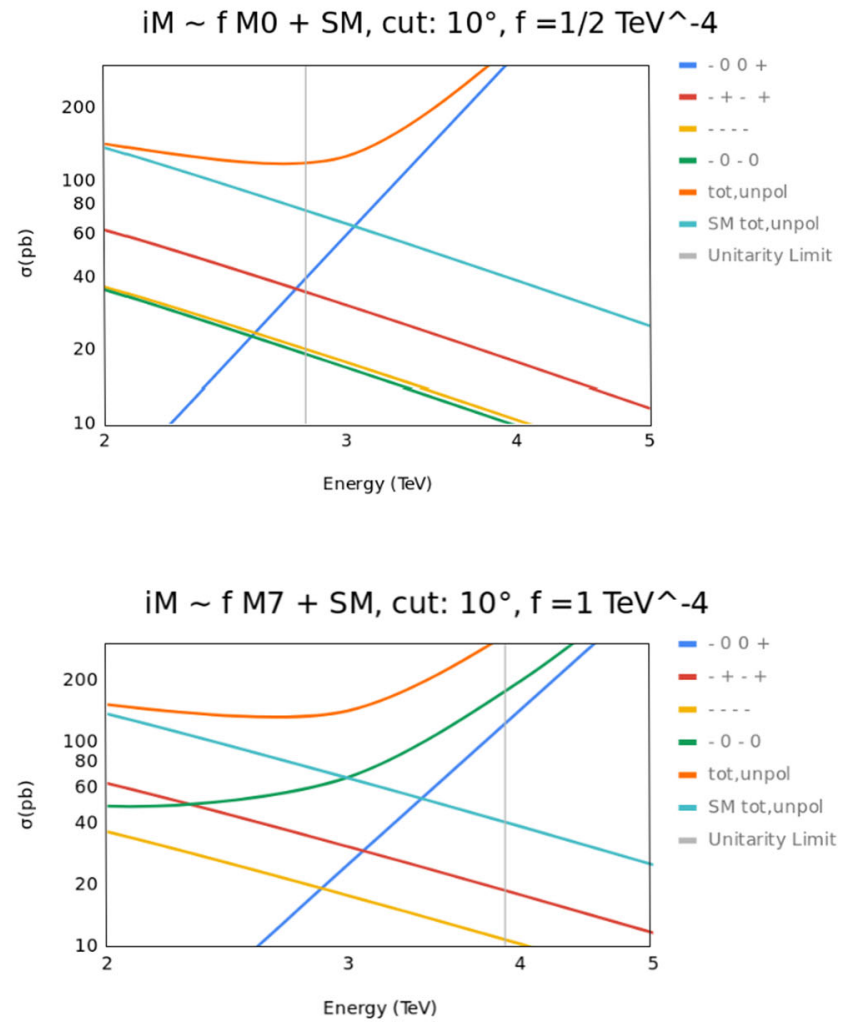

(not shown) polarized cross sections are negligibly small. In each plot shown is in addition the total cross section of a EFT "model" and the total cross section in the SM 

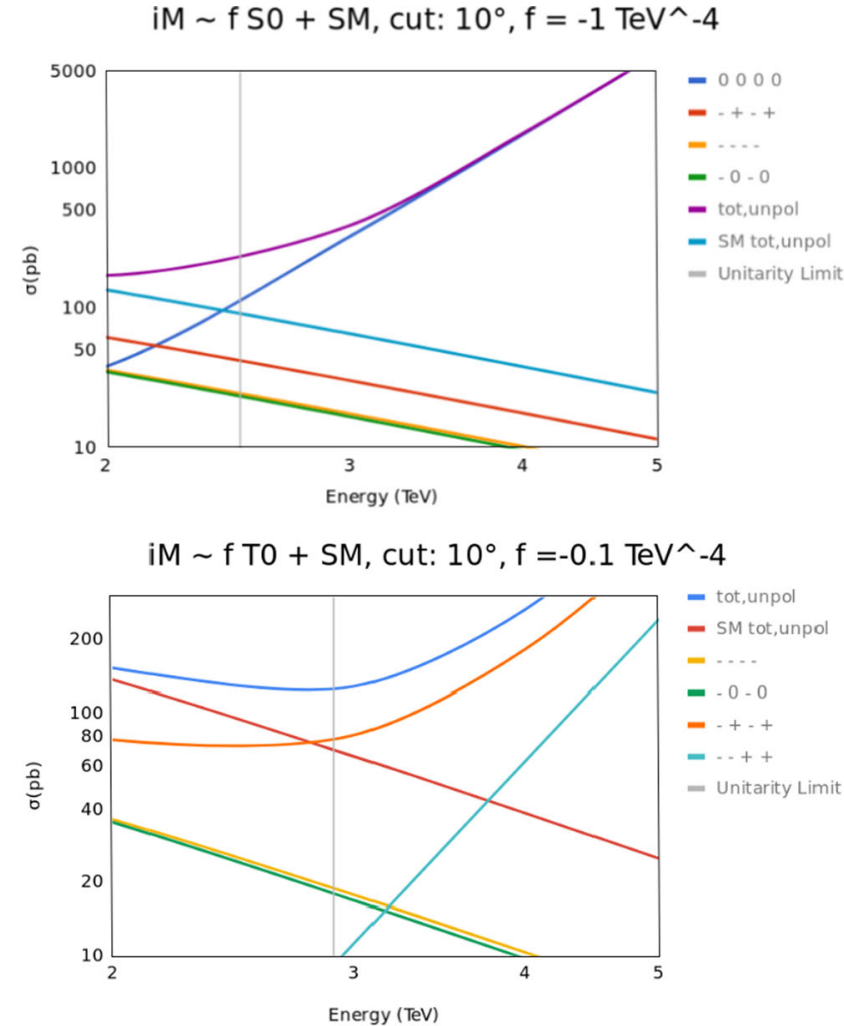

$\wedge-4$

- tot,unpol

- SM tot,unpol

$-\ldots$.

$=-0.0$

$-\cdot+\cdot+$

$-\cdots++$

- Unitarity Limit

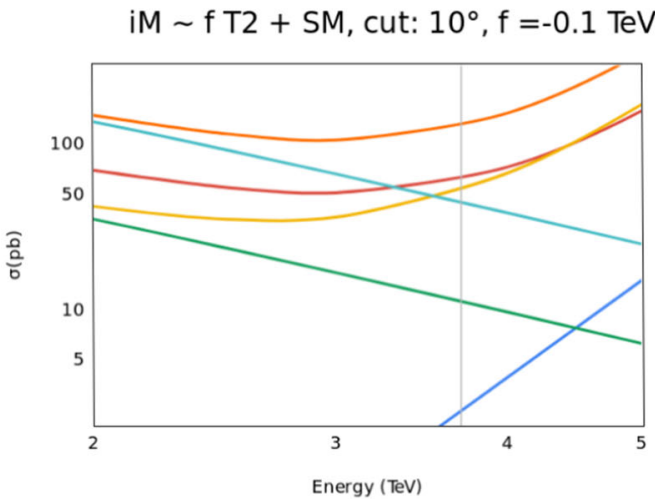

$\wedge$

$-\cdots++$

$-\cdot+\cdot+$

$-\cdots$

$--0-0$

- tot,unpol

- SM tot,unpol

- Unitarity Limit

$\mathrm{iM} \sim \mathrm{fM} 1+\mathrm{SM}$, cut: $10^{\circ}, \mathrm{f}=-1 \mathrm{TeV}^{\wedge}-4$

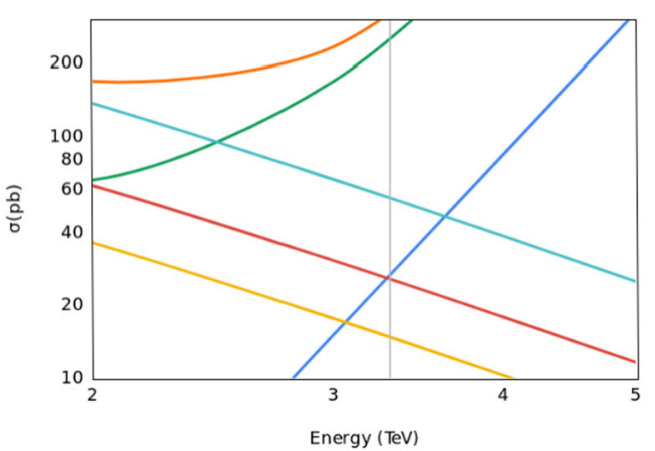

$-.00+$

$-\cdot+\cdot+$

$-\cdots$

- $-0-0$

- tot,unpol

- SM tot, unpol

- Unitarity Limit
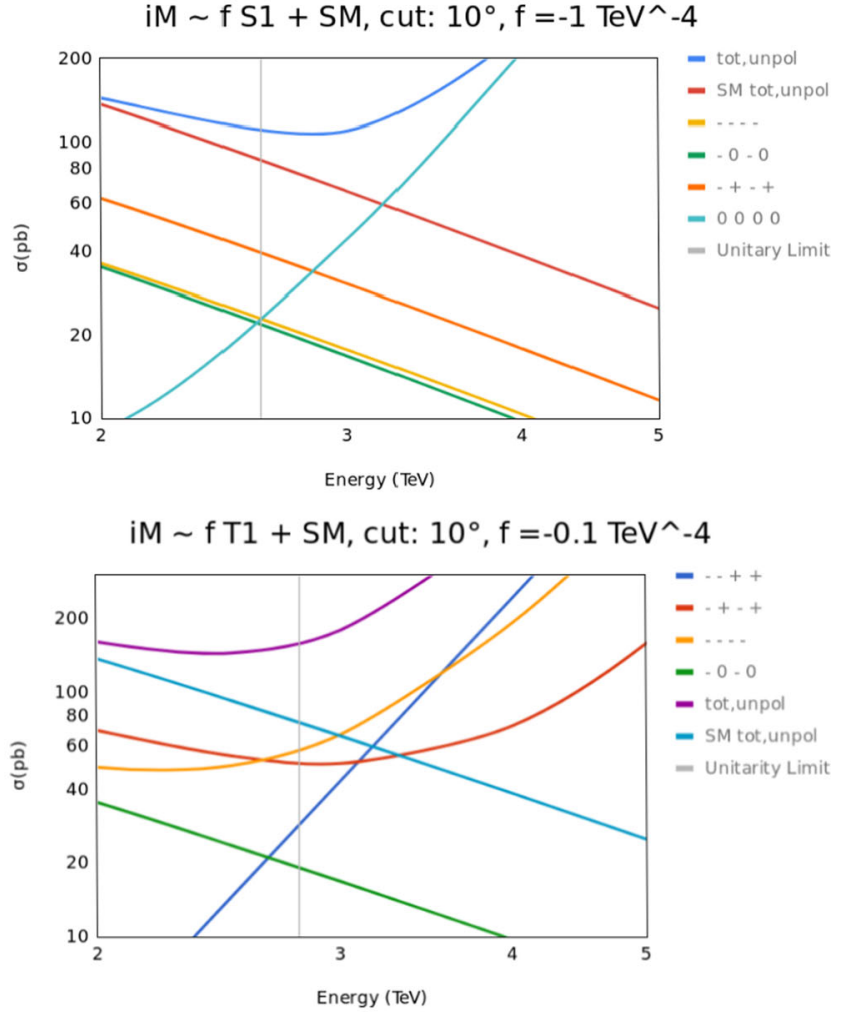

$\mathrm{iM} \sim \mathrm{fMO}+\mathrm{SM}$, cut: $10^{\circ}, \mathrm{f}=-1 / 2 \mathrm{TeV}^{\wedge}-4$

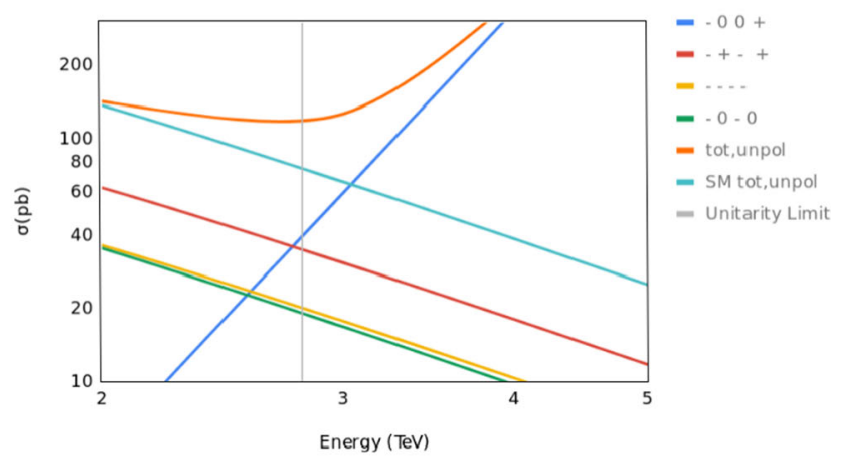

$\mathrm{iM} \sim \mathrm{f} \mathrm{M7} \mathrm{+} \mathrm{SM,} \mathrm{cut:} 10^{\circ}, \mathrm{f}=-1 \mathrm{TeV}^{\wedge}-4$

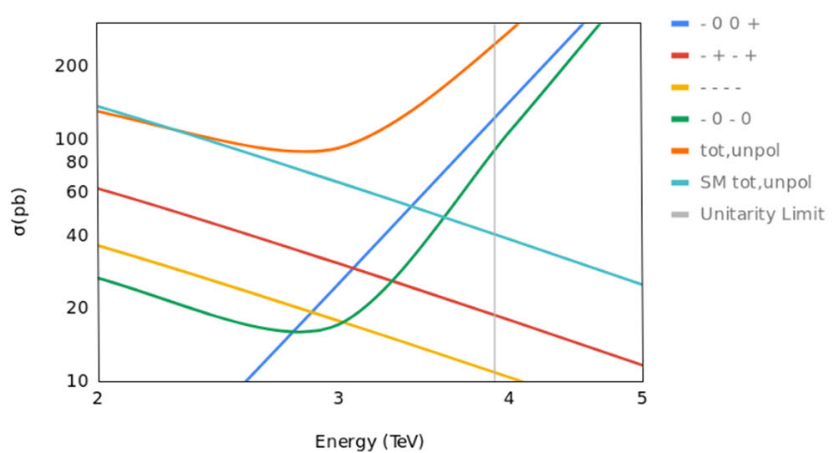

(not shown) polarized cross sections are negligibly small. In each plot shown is in addition the total cross section of a EFT "model" and the total cross section in the SM 
$\mathrm{iM} \sim \mathrm{f} \mathrm{SO}+\mathrm{SM}$, cut: $10^{\circ}, \mathrm{f}$ in $\mathrm{TeV}^{\wedge}-4$

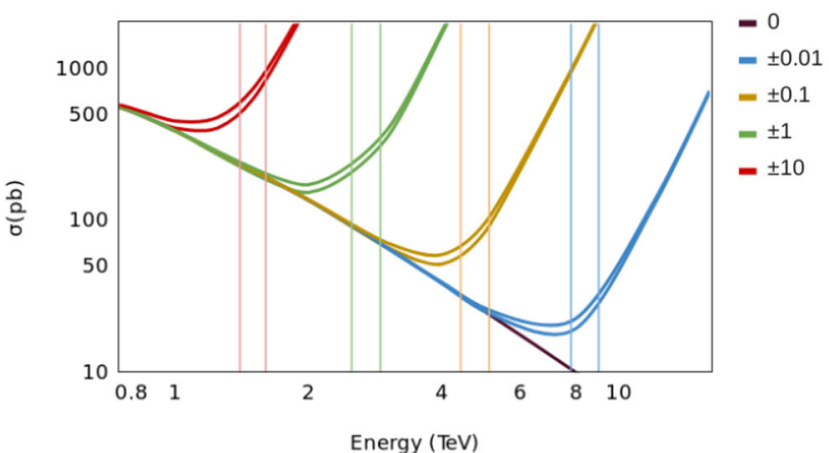

$i M \sim f T 0+S M$, cut: $10^{\circ}, f$ in $\mathrm{TeV}^{\wedge}-4$

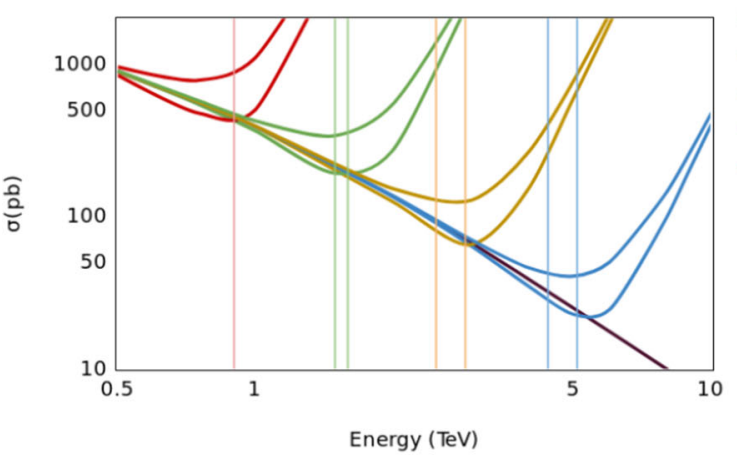

$\mathrm{iM} \sim \mathrm{fT} 2+\mathrm{SM}$, cut: $10^{\circ}, \mathrm{f}$ in $\mathrm{TeV}^{\wedge}-4$

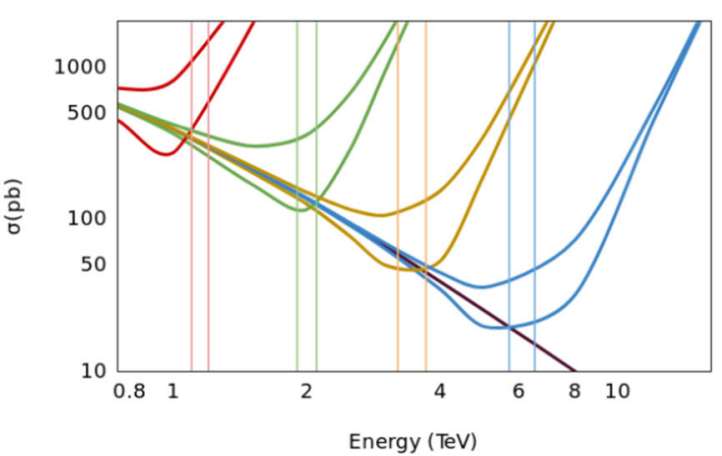

$\mathrm{iM} \sim \mathrm{fM}$ I + SM, cut: $10^{\circ}, \mathrm{f}$ in $\mathrm{TeV}^{\wedge}-4$

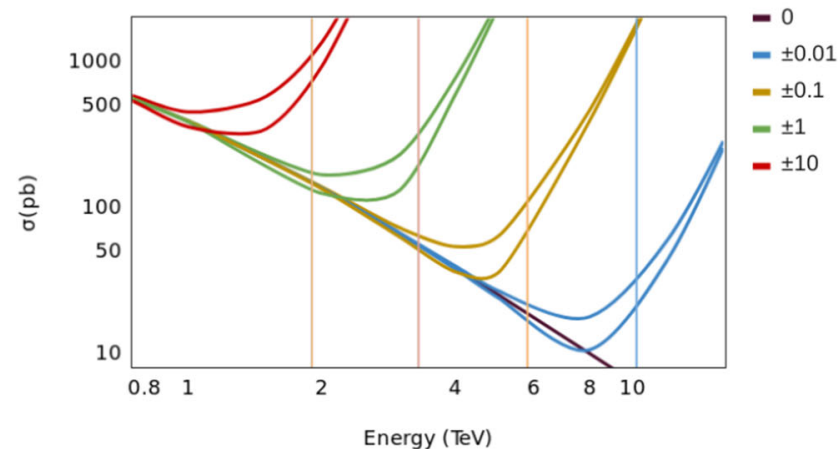

Fig. 8 Energy dependence of the total unpolarized $W^{+} W^{+}$cross sections $\left(E_{C M} \equiv \sqrt{s}\right.$, in TeV) for a chosen set of $f_{i}$ values. Vertical lines denote the unitarity bound $\sqrt{s^{U}}$ (color correspondence). There is no

$-0$

$= \pm 0.01$

$- \pm 0.1$

$- \pm 1$

$\mathbf{-} \pm 10$

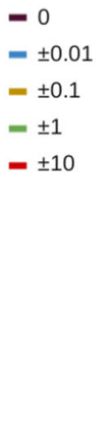

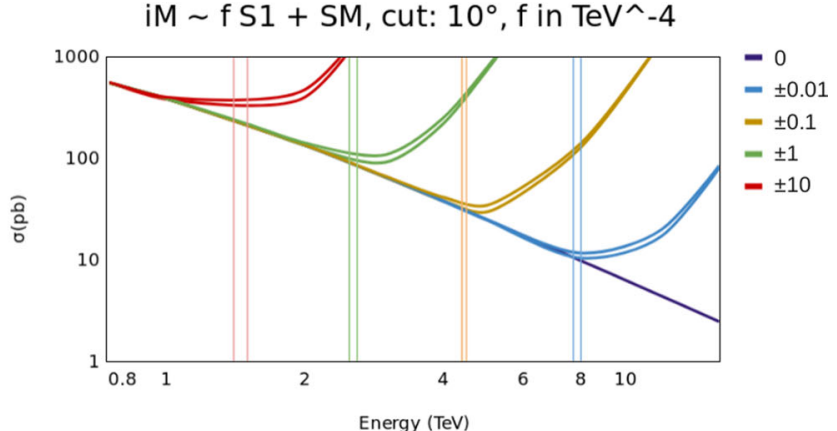

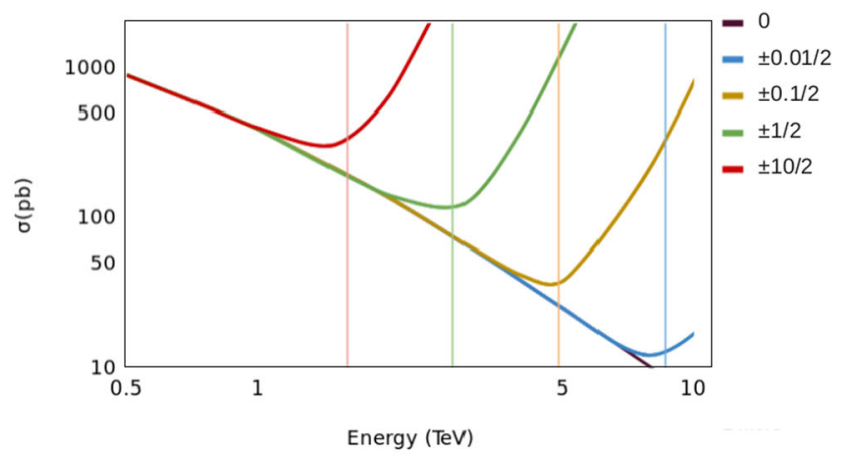

$i M \sim f M 7+S M$, cut: $10^{\circ}, \mathrm{f}$ in $\mathrm{TeV}^{\wedge}-4$

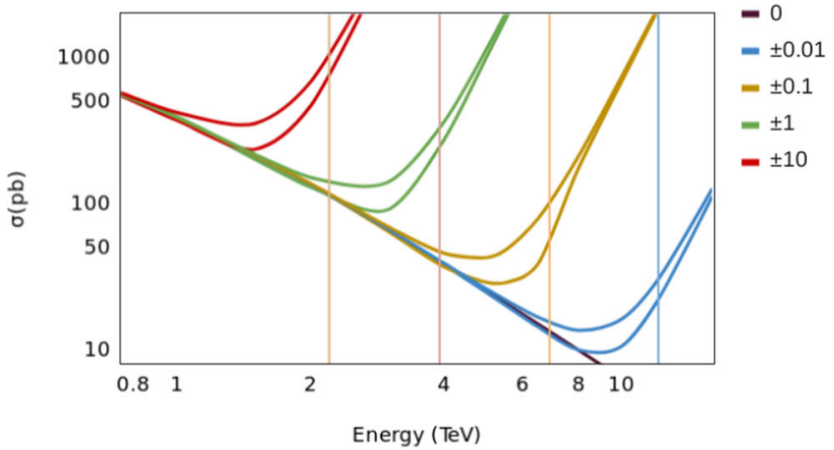

color distinction between the signs: except for $M 1$ and $M 7$, upper cross section curves correspond to $f<0$; in $S 0, S 1(T 0, T 2)$ stronger unitarity limits correspond to $f<0(f>0)$ 
Table 1 The helicity combinations yielding the strongest helicity partial waves unitarity limits for each operator in case of each sign of $f_{i}$. It is always a $\mathbf{J}=0$ partial wave that yields the strongest unitarity limits

\begin{tabular}{lllllllll}
\hline$i=$ & $S 0$ & $S 1$ & $T 0$ & $T 1$ & $T 2$ & $M 0$ & $M 1$ & $M 7$ \\
\hline$f_{i}>0$ & 0000 & 0000 & ---- and --++ & --++ & ---- & --00 & --00 & --00 \\
$f_{i}<0$ & 0000 & 0000 & --++ & --++ & --++ & --00 & --00 & --00 \\
\hline
\end{tabular}

derived from same-sign $W W$ partial wave expansion. This should be kept in mind in particular when using a VBFNLO calculator to determine the unitarity bounds that both sameand opposite-sign $W W$ scattering processes are looked at. The helicity combination yielding the strongest helicity partial wave unitarity limits for each operator are summed up in Table 1

\section{Extracting the BSM coupling from the discovery regions found}

Assuming that the departure form the SM predictions is indeed observed at the HL/HE-LHC we turn to the question what can be said about the couplings of higher dimension operators that defined the "EFT model". While the probed $\Lambda$ scale can be read off directly from Figs. 2, 3, the matching between fundamental parameters $C_{i}$ of a deeper BSM physics and the Wilson coefficients $f_{i}$ of the low energy approximation is needed to extract the information about couplings.

Let us start with operators that contain the stress tensor $W_{\mu \nu}$. The $W$ bosons, being fundamental $\mathrm{SU}(2)$ gauge bosons, would couple to the to-be-integrated-out BSM states via gauge coupling $g$. Therefore from the corresponding $f_{i}$ one can factor out $g^{2}$ for each $W_{\mu \nu}$. The Naive Dimensional Analysis (NDA) [17] suggests then the following matching

$$
\begin{aligned}
\mathcal{L} \supset f_{i} \mathcal{O}_{i} & \equiv c_{i} \cdot 2 \frac{g^{2}}{\Lambda^{4}} \mathcal{O}_{i}, \quad i=M 0, M 1 \\
f_{i} \mathcal{O}_{i} & \equiv c_{i} \cdot 2^{2} \frac{g^{4}}{16 \pi^{2} \Lambda^{4}} \mathcal{O}_{i}, \quad i=T 0, T 1, T 2 \\
f_{i} \mathcal{O}_{i} & \equiv c_{i} \cdot 2^{2} \frac{g^{2}}{\Lambda^{4}} \mathcal{O}_{i}, \quad i=M 6, M 7
\end{aligned}
$$

The factor 2 follows from the relation $\operatorname{Tr} \hat{W}_{\alpha \beta} \hat{W}_{\mu \nu}=$ $\frac{1}{2} W_{\alpha \beta}^{i} W_{\mu \nu}^{i}$ since in NDA the stress tensor $W_{\mu \nu}^{i}$ is used for counting purposes rather than the matrix form $\hat{W}_{\mu \nu}$. Extra factor 2 in case of $M 6, M 7$ operators is due to differences in the $\mathrm{SU}(2)$ structure, as can be seen from the relation $\mathcal{O}_{M 7}=\frac{1}{2} \mathcal{O}_{M 1}+\ldots$, while $\frac{1}{16 \pi^{2}}$ in front of $T$ operators is a single loop suppression factor suggested by the 4-th power of the electroweak coupling factored out.

The remaining dimensionless factors $c_{i}$ could be combinations of

$\frac{g_{*}}{4 \pi}, \quad \frac{y_{*}}{4 \pi}, \quad \frac{\lambda_{*}}{16 \pi^{2}}$,

where $g_{*}, y_{*}$ and $\lambda_{*}$ are some gauge, Yukawa and scalar couplings of the BSM sector of full theory, respectively. In the weakly-coupled theory one would expect that $c_{i}$ are naturally of order 1.

Using (C.1) it is straightforward to find the range of $c_{i}$ corresponding to the discovery regions shown in Figs. 2, 3.

\begin{tabular}{|c|c|c|c|c|c|c|}
\hline$f_{i}>0$ & $T 0$ & $T 1$ & $T 2$ & $M 0$ & $M 1$ & $M 7$ \\
\hline$c_{\min }-c_{\max }$ & $130-770$ & $120-1300$ & $670-2200$ & $23-32$ & $45-133$ & $33-140$ \\
\hline$f_{i}<0$ & $T 0$ & $T 1$ & & $T 2$ & $M 1$ & $M 7$ \\
\hline$c_{\min }-c_{\max }$ & $110-1500$ & $140-2600$ & & $410-4500$ & $48-130$ & 54-270 \\
\hline
\end{tabular}

Table 2 For each "EFT model" characterized by a choice of a single $n=8$ operator shown are the ranges of the overall coefficients $c_{i}$ in Eq. C. 1 that correspond to the discovery regions found in the $27 \mathrm{TeV}$ study

Table 3 See Table 2 for description; $14 \mathrm{TeV}$ case

\begin{tabular}{lllllll}
\hline$f_{i}>0$ & $T 0$ & $T 1$ & $T 2$ & $M 0$ & $M 1$ & $M 7$ \\
\hline$c_{\min }-c_{\max }$ & $137-790$ & $76-1300$ & $280-2200$ & $23-33$ & $38-140$ & $24-130$ \\
\hline$f_{i}<0$ & $T 0$ & $T 1$ & $T 2$ & $M 0$ & $M 1$ \\
\hline$c_{\min }-c_{\max }$ & $510-1400$ & $170-1200$ & $700-4100$ & $23-33$ & $45-140$ \\
\hline
\end{tabular}


The numerical values, presented in Tables 2 and 3 for 27 and $14 \mathrm{TeV}$ case, respectively, are found to be roughly consistent. However, instead of being of order 1 they are much larger. It suggests that in case of linearly realized spontaneous breaking of $S U(2) \times U(1)$ symmetry our method of probing BSM physics is sensitive only to strong dynamics. Alternatively one could consider relaxed assumptions concerning the symmetry breaking mechanism. For the analysis on "EFT triangles" in the so-called Higgs Effective Field Theory [18], see [19].

We turn now to the discussion of the $S 0$ and $S 1$ operators. We assume these are generated at loop level in the BSM. Otherwise they would come associated with $n=6$ operators, which are neglected in our analysis. Then, the NDA suggests

$\mathcal{L} \supset f_{i} \mathcal{O}_{i} \equiv c_{i} \cdot \frac{g_{*}^{4}}{16 \pi^{2} \Lambda^{4}} \mathcal{O}_{i}, \quad i=S 0, S 1$

Again, $c_{i}$ are some combinations of BSM couplings and naturally expected to be of order 1 . If we set $c_{i}=1$ in Eq. (C.3), then for $f_{S 0}>0$ we find that that $g_{*} \in(8.5 ; 10)$ and $g_{*} \in(8.4 ; 10)$ in the 14 and $27 \mathrm{TeV}$ case respectively. For $f_{S 0}<0$ we find $g_{*} \in(6.2 ; 8.9)$ and $g_{*} \in(7.3 ; 8.8)$ for the 14 and $27 \mathrm{TeV}$ case, respectively. The coupling is large, but interestingly it satisfies $g_{*}<4 \pi$. For $f_{S 1}$ the discovery regions are empty for both 14 and $27 \mathrm{TeV}$ cases for both signs.

\section{References}

1. J. Kalinowski, P. Kozów, S. Pokorski, J. Rosiek, M. Szleper, S. Tkaczyk, Eur. Phys. J. C 78, 403 (2018). https://doi.org/10.1140/ epjc/s10052-018-5885-y. arXiv:1802.02366 [hep-ph]

2. A. Alboteanu, W. Kilian, J. Reuter, JHEP 0811, 010 (2008). https:// doi.org/10.1088/1126-6708/2008/11/010. arXiv:0806.4145 [hep$\mathrm{ph}]$

3. W. Kilian, T. Ohl, J. Reuter, M. Sekulla, Phys. Rev. D 91, 096007 (2015). https://doi.org/10.1103/PhysRevD.91.096007. arXiv:1408.6207 [hep-ph]
4. O.J.P. Eboli, M.C. Gonzalez-Garcia, J.K. Mizukoshi, Phys. Rev. D 74, 073005 (2006). https://doi.org/10.1103/PhysRevD.74.073005. arXiv:hep-ph/0606118

5. C. Degrande et al., arXiv:1309.7890 [hep-ph]

6. J. Alwall et al., JHEP 07, 079 (2014). https://doi.org/10.1007/ JHEP07(2014)079. arXiv:1405.0301 [hep-ph]

7. T. Sjostrand, S. Mrenna, P. Skands, JHEP 0605, 026 (2016). https:// doi.org/10.1088/1126-6708/2006/05/026. arXiv:hep-ph/0603175

8. K. Arnold et al., Comput. Phys. Commun. 180, 1661 (2009). https:// doi.org/10.1016/j.cpc.2009.03.006. arXiv:0811.4559 [hep-ph]

9. J. Baglio et al., arXiv:1107.4038 [hep-ph]. arXiv:1404.3940 [hep$\mathrm{ph}]$

10. A. M. Sirunyan et al. [CMS Collaboration], Phys. Rev. Lett. 120(8), 081801 (2018). https://doi.org/10.1103/PhysRevLett.120.081801. arXiv:1709.05822 [hep-ex]

11. M. Aaboud et al. [ATLAS Collaboration], Phys. Rev. Lett. 123(16), 161801 (2019). https://doi.org/10.1103/PhysRevLett.123.161801. arXiv:1906.03203 [hep-ex]

12. C. Degrande, J. L. Holzbauer, S.-C. Hu, A. V. Kotwal, S. Li, M. Marx, O. Mattelaer, J. Metcalfe, M.-A. Pleier, C. Pollard, M. Rominsky, D. Wackeroth, arXiv:1309.7452 [physics.comp-ph]

13. B. Biedermann, A. Denner, M. Pellen, JHEP 1710, 124 (2017). https://doi.org/10.1007/JHEP10(2017)124. arXiv:1708.00268 [hep-ph]

14. A. Denner, M. Pellen, Section 4.2.2. in P. Azzi et al. [HL-LHC Collaboration and HE-LHC Working Group], arXiv:1902.04070 [hep-ph]

15. Q. Bi, C. Zhang, S.Y. Zhou, JHEP 1906, 137 (2019). https://doi. org/10.1007/JHEP06(2019)137. arXiv:1902.08977 [hep-ph]

16. A. Ballestrero, E. Maina, G. Pelliccioli, J. High Energy Phys. 2018, 170 (2018). https://doi.org/10.1007/JHEP03(2018)170. arXiv: 1710.09339 [hep-ph]

17. B.M. Gavela, E.E. Jenkins, A.V. Manohar, L. Merlo, Eur. Phys. J. C 76(9), 485 (2016). https://doi.org/10.1140/epjc/ s10052-016-4332-1. arXiv:1601.07551 [hep-ph]

18. R. Alonso, M. B. Gavela, L. Merlo, S. Rigolin, J. Yepes, Phys. Lett. B 722, 330 (2013). Erratum: [Phys. Lett. B 726, 926 (2013)]. https://doi.org/10.1016/j.physletb.2013.04.037. https://doi.org/10. 1016/j.physletb.2013.09.028. arXiv:1212.3305 [hep-ph]

19. P. Kozów, L. Merlo, S. Pokorski, M. Szleper, JHEP 1907, 021 (2019). https://doi.org/10.1007/JHEP07(2019)021. arXiv:1905.03354 [hep-ph] 